\title{
Simulation Study of Improving Mutative Scale Chaos Optimization Algorithmin Complex Nonlinear System
}

\author{
Zou En1,2, Huo Qing2,Huang Shuihong2,Chen Shengchuang1
}

(1. College of zhujiang. South China Agricultural University. Guangzhou 510900, P.R.China. 2.College of Engineer. South China Agricultural University. Guangzhou 510642, P.R.China.

\begin{abstract}
Keyword: Improving; Mutative scale; Chaos optimization; Complex nonlinear system.
\end{abstract}
\begin{abstract}
This paper addresses a reliability optimization problem, where the motive is to select the best optimization method for complex nonlinear system. In order to avoid blind and repeated searching of chaos optimization in searching space of complex nonlinear system, an improving mutative scale chaos optimization algorithm has been proposed to solve the problems. The algorithm counts better value for every searching and sets a sign $\mathrm{A}$ in the chaos searching, when the numbers of better value searched is equal to A, the searching space is dynamic reduced according scale, and the above course is repeated in the lesser scale till global optimal value is found. In order to check the reliability of the proposed solution methodology, five complex nonlinear functions have been simulated, the simulation results show that algorithm is simple and local searching ability is better, the efficiency is higher than that of mutative scale chaos optimization, and results demonstrate the benefits of the proposed algorithm for solving this type of problem.

Fund Project:Guangdong Province, province, the higher education innovation and strong school project (4724) project funding.
\end{abstract}

\section{I . INTRODUCTION}

To seek optimal parameters of control system, a number of exact methods have been proposed so far to deal with the combinatorial optimization problems, such as BP algorithm, gradient descent method, and so on, but they have disadvantages of slow convergence and tend to become trapped in local minimum. Simulate annealing method has been widely applied to various optimization problems too ${ }^{[1]}$, but which requires subtle adjustment of parameters in the annealing schedule such as the size of the temperature steps during annealing, the temperature range, the number of re-starts and re-direction of the search, etc. To design optimal parameters of system in detail is very difficult only by them.

Chaos optimization is an effective method that makes use of chaotic variables for optimal search. Using the features of ergodicity and randomness of chaotic motion does the search process; it can continually search for the optimal solution, and overcome the local minimum problem. Because the chaos search dynamics is confined in a relatively low-dimensional fractal space, compared to the stochastic search, which seems to realize an efficient search for a variety of optimization problems ${ }^{[2]}$. A chaos optimization algorithm has been proposed by literature [3], the optimal variables are transformed into chaotic variables by method of carrier-wave. It takes advantage of the intrinsic stochastic property and ergodicity of chaos movement to escape from the local minima, and direct optimization search within global range. But when search space is big, its search speed is too slow and effect is not good. A mutative scale chaos optimization method is proposed based on the chaos variables by literature [4], by continually reducing the searching space of variable optimized and enhancing the searching precision, the method made up lake of the literature, but it's step is complex and blind and repeated searching is not been avoided.

An improving mutative scale chaos optimization algorithm is proposed in base of literature[4]. The algorithm counts better value for every searching and sets a sign $\mathrm{A}$ in the chaos searching, when the numbers of better value searched is equal to $\mathrm{A}$, the searching space is dynamic reduced 
according scale, and the above course is repeated in the lesser scale till global optimal value is found. The method can improve search efficiency and local search ability, and its program is very simple.

\section{IMPROVING MUTATIVE SCALE}

\section{CHAOS OPTIMIZATION ALGORITHM}

On the global minimum optimization problem of continuous object,

$$
\begin{aligned}
& \min f\left(x_{i}\right) \\
& x_{i} \in\left[a_{i}, b_{i}\right], \quad i=1,2, \cdots, n
\end{aligned}
$$

In this paper optimization search is carried with Logistic mapping chaos variable.

$$
x_{n+1}=4 x_{n}\left(1-x_{n}\right)
$$

Steps of algorithm are as following:

Step1: Initialization. Giving $\mathrm{N}$ a larger circulation number and $\mathrm{M}$ a proper circulation number, the optimization variable range is as $\left[a_{i}, b_{i}\right]$;

Step2: Giving i different stochastically initialization values to $x_{i, 1}$ from $(0,1), x_{i}^{*}=x_{i, 1}$, the initialization time $=0$ and the initialization value of object function $f^{*}(x)$ as a larger number, then the better optimizing times are A;

Step3: For $m=1: \mathrm{M}_{\text {and }} n=1: \mathrm{N}$, mapping ${ }^{x_{i, n}}$ differently to corresponding defining field:

$$
x_{i, n}^{\prime}=a_{i}+x_{i, n}\left(b_{i}-a_{i}\right)
$$

Step4: carry ${ }^{x_{i, n}^{\prime}}$ to needed function and compare with it:

$$
\begin{aligned}
& \quad \text { if } f^{*}(x)=f\left(x_{i, n}^{\prime}\right), \\
& x_{i}^{*}=x_{i, n}^{\prime}, \quad \text { time }=\text { time }+1, \\
& \quad \text { if } \quad \text { time } \geq \mathrm{A} \quad \text { then go to Step7, otherwise continue. }
\end{aligned}
$$

Step5: carry $x_{i, n}$ to Logistic formula:

$$
x^{\prime \prime}{ }_{i, n}=4 x_{i, n}\left(1-x_{i, n}\right)
$$

Step6: giving $x_{i, n}=x_{i, n}, n=n+1$

$$
\text { if } n<\mathrm{N} \text { then go to Step3, }
$$

otherwise continue.

Step7: take the above $x_{i}^{*}$ as the approximation value of global optimization, and then reduce the optimizing range centered with it.

$$
\begin{aligned}
& a_{i}^{\prime}=x_{i}^{*}-\left(b_{i}-a_{i}\right) /(m+1) \\
& b_{i}^{\prime}=x_{i}^{*}+\left(b_{i}-a_{i}\right) /(m+1)
\end{aligned}
$$

( notes : If function is symmetrical on some point and all variables' optimizing range is same then reducing searching range centered with $x_{1}^{*}$ is a better way.

In order to assure searching ranges not to go beyond boundary make the restriction as followings:

$$
\begin{aligned}
& \text { if } a_{i}^{\prime}<a_{i} \text {, then } a_{i}^{\prime}=a_{i} \text {, } \\
& \text { if } b_{i}^{\prime}>b_{i} \text {, then } b_{i}^{\prime}=b_{i}
\end{aligned}
$$


Step8: $m=m+1$

if $m<\mathrm{M}$, then give $a_{i}=a_{i}^{\prime}, b_{i}=b_{i}^{\prime}$,

return step3, otherwise continue.

Step9: when the suspending searching condition is meted, the optimization parameter $x_{i}^{*}$ is gained from minimum of $f^{*}(x)$

\section{EXAMPLES}

By way of compare, the five complex testing functions are simulated.

$$
\begin{gathered}
F_{1}=100\left(x_{1}^{2}-x_{2}\right)^{2}+\left(1-x_{1}\right)^{2}, \\
-2.048 \leq x_{1}, x_{2} \leq 2.048 \\
F_{2}=\left(4-2.1 x_{1}^{2}+x_{1}^{4} / 3\right) x_{1}^{2}+x_{1} x_{2}+\left(-4+4 x_{2}^{2}\right) x_{2}^{2}, \\
\quad-100<x_{1}, x_{2}<100 \\
F_{3}=-0.5+\left(\sin ^{2}\left(\sqrt{x_{1}^{2}+x_{2}^{2}}\right)-0.5\right) /\left(1+0.001\left(x_{1}^{2}+x_{2}^{2}\right)\right)^{2}, \\
\quad-100<x_{1}, x_{2}<100 \\
F_{4}=\left(x_{1}^{2}+x_{2}^{2}\right)^{0.25}\left[\sin ^{2}\left(50\left(x_{1}^{2}+x_{2}^{2}\right)^{0.1}+1\right],\right. \\
\quad-100<x_{1}, x_{2}<100 \\
F_{5}=\left[1+\left(x_{1}+x_{2}+1\right)^{2}\right. \\
\left.\quad\left(19-14 x_{1}+3 x_{1}^{2}-14 x_{2}+6 x_{1} x_{2}+3 x_{2}^{2}\right)\right] \\
\cdot\left[30+\left(2 x_{1}-3 x_{2}\right)^{2}\right. \\
\left.\quad\left(18-32 x_{1}+12 x_{1}^{2}+48 x_{2}-36 x_{1} x_{2}+27 x_{2}^{2}\right)\right] \\
\quad-2 \leq x_{1}, x_{2} \leq 2
\end{gathered}
$$

According the methods have been used of the literature [3], the literature [4] and this paper, stochastic select initial values in $(0,1)$ range to simulating, the simulated results are shown in Tab.

TABLE 1 The compare of methods this paper and literature $[3,4]$

\begin{tabular}{|l|c|c|c|c|c|}
\hline \multirow{2}{*}{ function } & \multirow{2}{*}{$\begin{array}{l}\text { Optimal } \\
\text { value }\end{array}$} & \multirow{2}{*}{$\begin{array}{c}\text { global } \\
\text { extremum }\end{array}$} & & \multicolumn{3}{|c|}{$\begin{array}{l}\text { optimize time (S) } \\
\text { literature [3] }\end{array}$} & $\begin{array}{l}\text { arithmetic of } \\
\text { literature [4] }\end{array}$ & $\begin{array}{l}\text { arithmetic of } \\
\text { this paper }\end{array}$ \\
\hline$F_{1}$ & $(1.1)$. & 0 & 0.9257 & 0.8574 & 0.3600 \\
\hline$F_{2}$ & $(-0.089,0.712)$ & -1.031 & 8.3562 & 0.7774 & 0.6210 \\
& $(0.089,-0.712)$ & & & & \\
\hline$F_{3}$ & $(0,0)$ & -1 & 52.793 & 6.6849 & 1.6820 \\
\hline$F_{4}$ & $(0,0)$ & 0 & 225.5385 & 22.3115 & 3.3050 \\
\hline$F_{5}$ & $(0,-1)$ & 3 & 15.6388 & 3.9731 & 1.6420 \\
\hline
\end{tabular}

As discussed in the above table I shows that the optimize time of this paper is little compare with that of literature [3] and [4] in time. 


\section{SIMULATION STUDY}

Simulate to the above five complex test functions in each variable range [ $\left.{ }_{i}, b_{i}\right]$, the results are shown in Fig. $1 \sim$ Fig.5. Abscissa is time of search, "**)is value has been searched, as $F_{1} 、 F_{2}$ and $F_{5}$ are too convergence in the figure, only local function values are lined out.

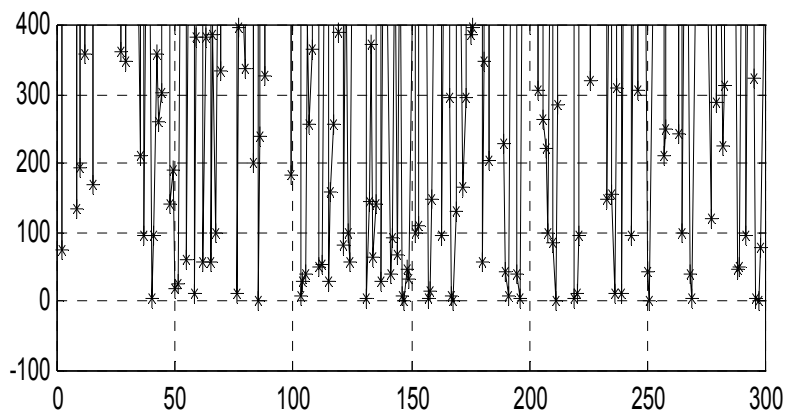

Fig.1 Portrait local function value of $F_{1}$ at chaos search 300 time

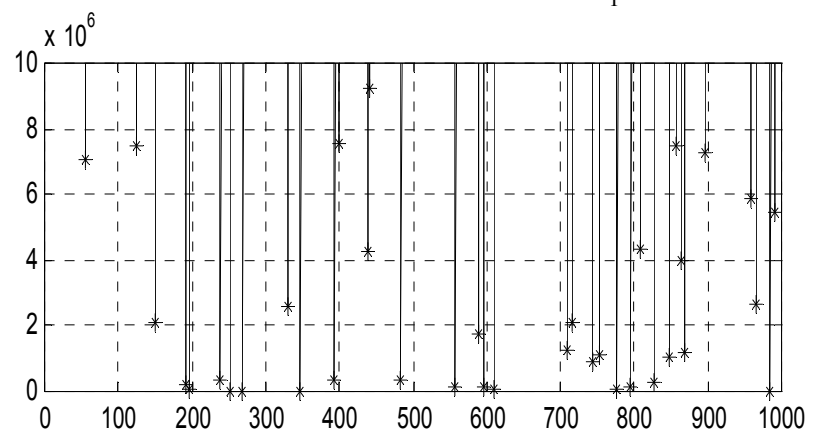

Fig.2 Portrait local function value of $F_{2}$ at chaos search 1000 time

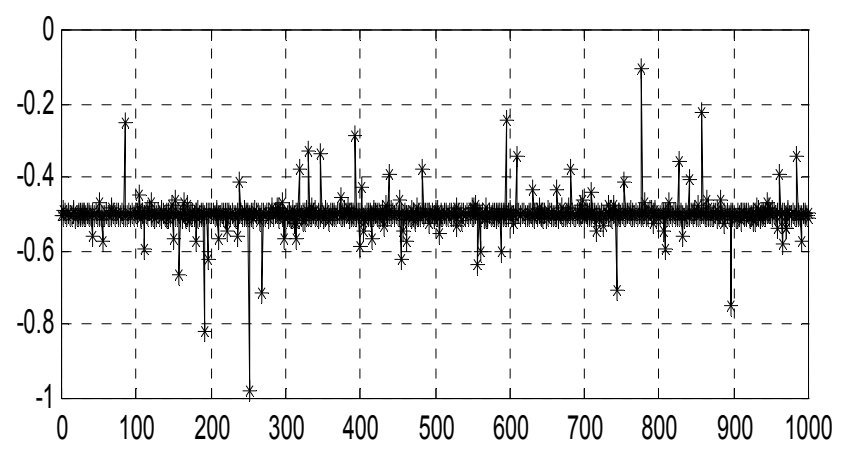

Fig.3 Chaos search function value of $F_{3}$

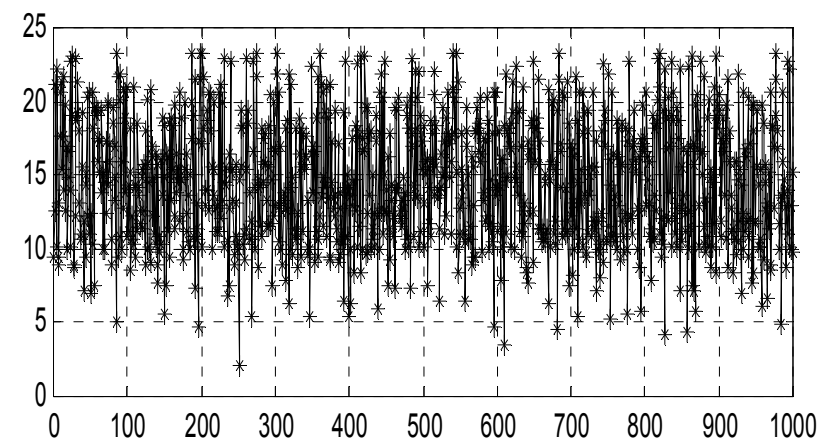

Fig.4 Chaos search function value of $F_{4}$ 


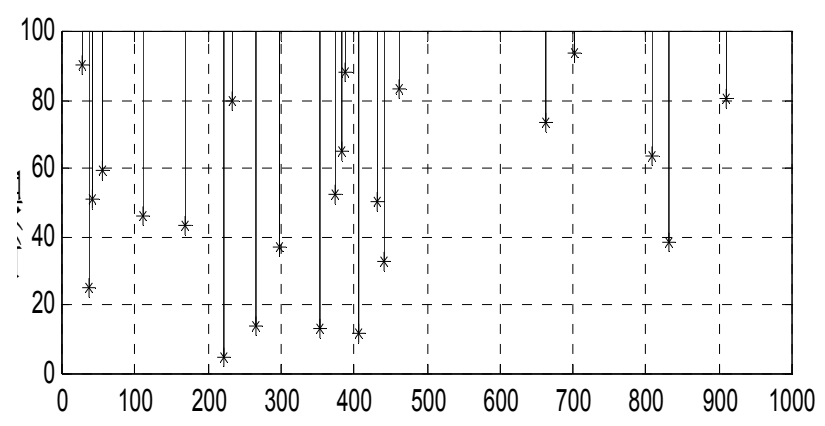

Fig.5 Portrait local function value of $F_{5}$ at chaos search 1000 time

Seeing from Figure, currently optimal value is discovered of small range function $F_{1}$ and $F_{5}$ in compare to three times, currently optimal value is discovered of big range function $F_{2}$ and $F_{4}$ in compare to five and six times, and that is discovered of complex function $F_{3}$ in compare to seven times. My experience is that $\mathrm{N}$ is equal to $1000, \mathrm{~A}$ is equal to $5 \sim 7, \mathrm{M}$ is equal to $20 \sim 30$ when search space is big, and $\mathrm{A}$ is equal to $3 \sim 5, \mathrm{M}$ is equal to $10 \sim 20$ when search space is small.

\section{V.ASTRINGENCY PROOF}

Definition 1 Suppose $x_{n}, n \in(1,2, \cdots, N)$ are chaos sequence is defined in chaos dynamic system, if chaos variable $x$ exist, and $\forall \varepsilon>0$, as while as

$\lim _{n \rightarrow \infty} \mathrm{P}\left(\left|x_{n}-x\right|<\varepsilon\right)=1$

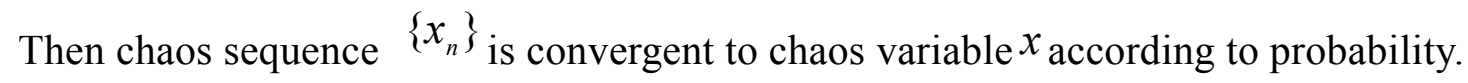

Definition 2 On above chaos sequence ${ }^{x_{n}}$ and chaos variable $x$,

$$
\text { If } \quad \mathrm{P}\left\{\lim _{n \rightarrow \infty}\left(x_{n}-x\right)=0\right\}=1
$$

or if $\forall \varepsilon>0$, as will as

$$
\mathrm{P}\left\{\bigcap_{n=1}^{\infty} \bigcup_{i \geq n}\left(\left|x_{i}-x\right| \geq \varepsilon\right)\right\}=0
$$

Then chaos sequence $\left\{x_{n}\right\}$ can be convergent to chaos variable $x$ according to probability 1 . According to definition 1and 2, we can gained theorem is shown as fellows.

Theorem Giving sequence produced by function $f(x)$ with chaos algorithm as $\left\{x_{n}\right\}$ then $\min f(x)$ can be convergent to global optimal value $x^{*}$ based on probability 1 .

Prove suppose $x_{\mathrm{i}}^{*}=\arg \min _{x \in \mathrm{N}} f(x), \mathrm{N} \in \mathrm{R}^{\mathrm{n}}$

Giving $\varepsilon$ 's small range of global optimal value $x^{*}$ as ${ }^{N_{\varepsilon}}$, then for any positive $\varepsilon, \delta>0$ exists and meets the following:

$$
N_{\varepsilon}=\left\{x|| x-x^{*}|<\delta,| f(x)-f\left(x^{*}\right) \mid<\varepsilon\right\}, \quad x \in \mathrm{R}^{i}
$$

The random sequence embedded in $N_{\varepsilon}$ is as followings:

$$
\mathrm{A}_{k}=\left\{x \mid x_{i, n}^{*} \in N_{\varepsilon}, n \in\{1,2, \cdots, \mathrm{N}\}\right\}
$$

It stands for iterative sequence going into $x^{*}$, s near field $\mathrm{N}_{\varepsilon}$ nth. Because chaos optimization algorithm is a descend algorithm mechanism, $f\left(x_{i, n}\right)$ is a monotony descend sequence by all appearances. So exists:

$$
f\left(x_{i, 1}\right)>f\left(x_{i, 2}\right)>\cdots>f\left(x_{i, n}\right)>\cdots>f\left(x^{*}\right)
$$


It makes out that $x_{\varepsilon}$ is a searching field. Accordingly as long as the parameter $x_{i, i}$ goes into $_{\tau} x_{\tau}$ the parameter ${ }^{x_{i, i+1}}$ goes into $x_{\varepsilon}$ certainly. Namely when event $\mathrm{A}_{1}$ occurs event $\mathrm{A}_{2}$ occurs certainly, it causes occurring of event ${ }^{A}$ certainly, ..., so exists:

$\mathrm{A}_{1} \subset \mathrm{A}_{2} \subset \cdots \subset \mathrm{A}_{n} \subset \cdots \cdots$

It also accounts for probability being monotony ascend.

$\mathrm{P}\left(\mathrm{A}_{1}\right) \leq \mathrm{P}\left(\mathrm{A}_{2}\right) \leq \cdots \leq \mathrm{P}\left(\mathrm{A}_{n}\right) \leq \cdots$

And $0 \leq \mathrm{P}\left(\mathrm{A}_{n}\right) \leq 1$, Based on the principle that monotony ascends series with upper boundary is of limit certainly, random accident series $\mathrm{A}_{n}(n=1,2 \cdots)$ is convergent on probability:

$$
\lim _{n \rightarrow \infty} \mathrm{P}\left(\mathrm{A}_{n}\right)=1
$$

\section{CONCLUSIONS}

According to above discussions and studies, the optimization algorithm expatiated in this paper has remedied the shortage of paper [4]. Not only has the chaos optimizing process avoided overlap searching but can dynamically reduce searching space according process .This algorithm can over the local minimum and enhance local searching ability. It has the feature of global optimization and the simple algorithm's construct .The algorithm is easy to realize and of high searching efficiency.

\section{Acknowledgment}

This work was financially supported by Guangdong Province, province, the higher education innovation and strong school project (4724).

\section{REFERENCES}

[1]Luonan Chen,Kazuyuki Aihara. Chaotic simulated annealing by a neural network model with transient chaos[J].Neural Networks, 1995,8(6):915-930.

[2] Zou En,Li Xiangfei,Chen Jianguo.Chaotic control and optimization applications[M]. Changsha: National University of Defense Technology Press,2002 (in Chinese)

[3] LI Bing and JIANG Weisun. Chaos Optimization Method and Its Application[J]. CONTROL THEORY \& APPLICATIONS,1997, 14(4): 613- 615.

[4] Zhang Tong, Wang Hongwei; Wang Zicai. Mutative Scale Chaos Optimization Algorithm and Its Application [J]. Control and Decisioy 1999, 14(3): 285-288. 